\title{
Evaluation of a semiquantitative SNAP test for measurement of bile acids in dogs
}

Background: Serum bile acids (SBA) are used as a routine screening tool of liver function in dogs. Serum samples are usually shipped to a referral laboratory for quantitative analysis with an enzymatic chemistry analyzer. The canine SNAP Bile Acids Test (SNAP-BAT) provides an immediate, semi-quantitative measurement of bile acid concentrations in-house. With the SNAP-BAT, bile acids concentrations of 5 to $30 \mu \mathrm{mol} / \mathrm{L}$ are quantified, and results outside of that range are classified as $<5$ or $>30 \mu \mathrm{mol} / \mathrm{L}$. Agreement of the SNAP-BAT with the enzymatic method has not been extensively investigated. Objectives: The purposes of this prospective clinical study were to assess the precision of the SNAP-BAT and determine agreement of SNAP-BAT with results from an in-house chemistry analyzer. Methods: After verifying intra-assay precision of the SNAP-BAT, a prospective analysis was performed using blood samples collected from 56 dogs suspected to have liver disease. Each sample was analyzed with an enzymatic, in-house chemistry analyzer and the SNAP-BAT. Agreement between the two methods was statistically assessed using the $\mathrm{k}$ index of agreement.

Results: Intra-assay variability was minimal. The $\mathrm{K}$ index for agreement between the SNAPBAT and routine chemistry analyzer was between $0.752-0.819$, indicating substantial to near perfect agreement.Conclusions: The SNAP-BAT is a highly accurate, semi-quantitative test that yields immediate results, and has very little intra-assay variability, particularly for results $>30 \mu \mathrm{mol} / \mathrm{L}$. 


\section{Evaluation of a semiquantitative SNAP test for measurement of bile acids in dogs}

2 Rachel L. Seibert, Karen M. Tobias, Ann Reed, Karl R. Snyder

3 Department of Small Animal Clinical Sciences, College of Veterinary Medicine, University of Tennessee,

4 Knoxville, TN 37996-4544

5 Corresponding author: Rachel L. Seibert, rseiber2@utk.edu 


\section{Introduction}

7 Bile acids are synthesized exclusively within hepatocytes from cholesterol, then excreted into the

8 biliary tract and stored in the gallbladder. After a meal, bile acids are released into the duodenum,

9 where they facilitate lipid digestion and absorption. Over $95 \%$ of bile acids are reabsorbed in the

10 distal ileum and jejunum and transported, via the portal circulation, to the liver. Within the liver,

11 bile acids are removed from portal blood by hepatocytes and recycled back into the biliary system

12 (termed enterohepatic circulation). An increase in SBA can therefore be expected with hepatic,

13 biliary, or portal disorders that limit hepatic portal blood flow or hepatocellular uptake. ${ }^{1}$ In dogs,

14 pre- and postprandial SBA have repeatedly been found to be reliable markers for the diagnosis of

15 hepatic and biliary dysfunction and disorders of the portal vasculature, such as portosystemic

16 shunts (PSS) and congenital portal vein hypoplasia with secondary microvascular dysplasia

17 (PVH-MVD). In previous studies of dogs with PSS, sensitivity of increased SBA concentration

18 for diagnosis of PSS ranged from $64 \%$ to $100 \%{ }^{2-5}$ The specificity of SBA for the diagnosis of

19 liver disease exceeds $90 \%$ at SBA concentrations greater than or equal to $30 \mu \mathrm{mol} / \mathrm{L}$ and reaches

$20100 \%$ at concentrations greater than or equal to $50 \mu \mathrm{mol} / \mathrm{L} .{ }^{6}$

21 The clinical standard for measurement of bile acids is considered to be an enzyme-based assay,

22 which provides fully quantitative results. However, most veterinary practices do not have this

23 methodology available in-house. Samples must be sent off to an outside laboratory, resulting in

24 delays in diagnosis and treatment. Recently, a rapid, in-house bile acid test- the IDEXX Canine

25 Snap Bile Acids Test [SNAP-BAT] ${ }^{\text {a }}$ - has become commercially available. The SNAP-BAT is a

26 semi-quantitative, competitive immunoassay that fully quantifies values of 5 to $30 \mu \mathrm{moles} / \mathrm{L}$ and

27 yields semiquantitative results for bile acids $<5 \mu$ moles/L and $>30 \mu$ moles $/ \mathrm{L}$. This test measures

28 the concentration of bile acids using an antibody present within the reagent (or conjugate), which

29 is mixed with the serum sample. The conjugate contains an antibody to bile acids with an enzyme 
30 linked to it (termed "labeled"), which allow for detection of bile acids by producing an

31 observable color change when the labeled antibody binds to bile acids. Because this is an in-

32 house test, the SBAT is becoming more widely used by private practitioners as a screening test

33 for dogs with clinical signs consistent with portal vascular anomalies and other liver diseases. In

34 a method comparison study by the manufacturer, overall agreement of the SNAP-BAT with the

35 reference assay (Hitachi Chemistry Analyzer) was $96.2 \%$ for bile acid concentrations $<12 \mu \mathrm{mol} / \mathrm{L}$

36 and $95.2 \%$ for bile acid concentrations $>25 \mu \mathrm{mol} / \mathrm{L} .{ }^{7}$ However, statistical assessment of

37 agreement between the two tests and calculation of test sensitivity and specificity for diagnosis of

38 liver dysfunction were not performed in that study. Additionally, evaluation of the new SNAP-

39 BAT was performed by the manufacturer; independent analysis by a third party has not been

40 reported.

41 The purposes of this prospective clinical study were to assess the precision, or intra-assay

42 variability, of the SNAP-BAT and determine agreement of SNAP-BAT with results from a

43 standard in-house enzymatic chemistry analyzer ${ }^{b}$. We hypothesized intra-assay variability of the

44 SNAP-BAT would be minimal and there would be no statistically significant difference in results

45 of SBAT and the in-house, enzymatic chemistry analyzer for quantitative and semiquantitative

46 data.

\section{Materials and Methods}

$48 \underline{\text { Samples }}$

49 All procedures were approved by the University of Tennessee Institutional Animal Care and Use 50 Committee (Number: 2053-1011) before commencement of the study, and informed consent was 51 obtained from owners of all enrolled

52 dogs. Analysis was performed on blood drawn for enzymatic bile acid analysis from patients at

53 the University of Tennessee or serum submitted by local practices to the University of Tennessee

54 Clinical Pathology Laboratory. Samples obtained in-house were classified as preprandial or 
55 postprandial. Preprandial samples were obtained after a fast of 12 or more hours, and

56 postprandial samples were obtained 2 hours after feeding. Samples were collected by cephalic,

57 saphenous, or jugular venipuncture from in-house patients. Blood was placed in a red top tube

58 containing no additives, allowed to clot for a minimum of 20 minutes, and then centrifuged for 10

59 minutes at $2250 \mathrm{~g}^{\mathrm{c}}$. The serum was then separated and stored at $-20^{\circ} \mathrm{C}$ until the time of analysis.

60 Because specific fasting and feeding information was not available, samples submitted from local

61 practices were considered unclassified. Serum samples submitted by local practices were also

62 similarly centrifuged, separated from any remaining red cells, and stored at $-20^{\circ} \mathrm{C}$. Maximum

63 storage time was 3 months.

\section{Sample Analysis}

65 At the time of biochemical analysis, frozen samples were thawed at room temperature and gently

66 agitated until all crystals were dissolved. All SNAP-BAT were performed by the same evaluator

67 (RS) using the methodology described by the manufacturer ${ }^{\text {a }}$ with interpretation of colorimetric

68 changes provided by the SNAP Reader. ${ }^{\text {a }}$ The standard enzymatic chemical analysis was

69 performed by the same clinical pathology technician (KS).

70 Intra-assay variability: Five frozen serum samples were randomly selected, thawed as described

71 above, and combined into a pooled sample. Each pooled sample was evaluated with the standard

72 chemistry analyzer ${ }^{\mathrm{b}}$ using an enzyme-based reagent. ${ }^{\mathrm{d}}$ Each pooled sample was then divided into

7310 allotments, and each allotment was evaluated with a SNAP-BAT. This process was repeated 4

74 more times to generate a total of 5 pooled samples, resulting in a total of 50 SNAP-BAT and 5

75 standard, enzymatic chemistry analyzer tests. All sample analyses were performed on the same

76 day to reduce testing variables. 
77 Quantitative analysis: After confirmation of minimal intra-assay variability, quantitative analysis

78 was performed on samples different from those used for the intra-assay variability testing. Frozen

79 serum samples were thawed as described above. One aliquot $(0.5 \mathrm{ml})$ from each sample was

80 evaluated using the standard, enzymatic chemistry analyzer. The remaining serum from each

81 sample was evaluated on the same day using the SNAP-BAT.

82 Statistical analysis:

83 For general agreement, bile acid concentrations measured by SNAP-BAT assay were compared

84 with the results of enzymatic analysis using the $\kappa$ index. ${ }^{8,9}$ Reference range for normal bile acids

85 was set at 0 to $20 \mu \mathrm{mol} / \mathrm{L}$, which is the reference range currently used by our laboratory for

86 results of enzymatic analysis. This reference range was used regardless of whether the sample

87 was pre- or postprandial ${ }^{10}$. Calculations of the $\kappa$ index' sensitivity, specificity, and negative and

88 positive predictive values of the SNAP-BAT were therefore calculated based on a cutoff value of

$8920 \mu \mathrm{mol} / \mathrm{L}$. Data analysis was performed using two groupings: Group 1, which included results

90 from dogs with postprandial samples and those with a single, unclassified serum sample; and

91 Group 2, which included results from preprandial samples and dogs with a single, unclassified

92 serum sample. Each analysis therefore included one observation per dog to avoid

93 pseudoreplication of results.

94 Each analysis was further divided into two sets of data: bile acid concentrations $\leq 20 \mu \mathrm{mol} / \mathrm{L}$ or $>$

$9520 \mu \mathrm{mol} / \mathrm{L}$, and bile acid concentrations $<5 \mu \mathrm{mol} / \mathrm{L}, 5-30 \mu \mathrm{mol} / \mathrm{L}$, and $>30 \mu \mathrm{mol} / \mathrm{L}$. Because

96 variances were not equal, the nonparametric Mann-Whitney test was used to test differences in

97 enzymatic and SNAP-BAT results using two SNAP categories (SNAP-BAT $\leq 20 \mu \mathrm{mol} / \mathrm{L}$; SNAP-

98 BAT $>20 \mu \mathrm{mol} / \mathrm{L})$. The Kruskal-Wallis test was used to compare three categories of results

99 (SNAP-BAT $<5 \mu \mathrm{mol} / \mathrm{L} ; 5-30 \mu \mathrm{mol} / \mathrm{L}$, and $>30 \mu \mathrm{mol} / \mathrm{L}$ ) with those of the enzymatic results for

100 the same sample. A P value $<0.05$ was used to indicate statistical significance. 
102 Samples from 25 dogs were used for evaluation of intra-assay variability. Intra-assay variability

103 was minimal overall, with no variability seen in samples with values $>30 \mu \mathrm{mol} / \mathrm{L}$ (Table 1 ).

104 Because of lack of variability and small sample size, statistical analysis of these results was

105 unable to be performed.

106 Samples from 56 dogs were included in the quantitative analysis. Paired (pre- and postprandial)

107 bile acid samples were available from $17 \mathrm{dogs}$, and a single, unclassified sample was available

108 from each of 39 dogs. The cross tabulation data for results of enzymatic chemistry analysis and

109 SNAP-BAT for Group 1 and Group 2 are reported in Tables 2 and 3, respectively. For Group 1

110 results, sensitivity of the SNAP-BAT was $100 \%$ and specificity was $78.6 \%$. The positive

111 predictive was $82.4 \%$, while the negative predictive value was $100 \%$. For Group 2 results,

112 sensitivity of SNAP-BAT was $100 \%$ and specificity was $83.9 \%$. The positive predictive value

113 was $83.3 \%$ and negative predictive value was $100 \%$.

114 For Group 1 samples, the $\kappa$ index for agreement between the SNAP-BAT and routine chemistry

115 analyzer was 0.786 . For Group 2 samples, the $\kappa$ index for agreement between the SNAP-BAT and

116 routine chemistry analyzer was 0.819 (Table 4 ). When enzymatic chemistry values were ranked

117 based on two SNAP-BAT categories $(\leq 20$ and $>20 \mu \mathrm{mol} / \mathrm{L})$ using the Mann-Whitney test, the

118 values differed significantly from each other between the two categories for both Group 1 and

119 Group 2 (Mann-Whitney $U=31.5, p<0.001$ for Group 1; Mann-Whitney $U=25.5, p<0.001$ for

120 Group 2). Similarly, when enzymatic chemistry values were ranked based on three SNAP-BAT

121 categories $(<5 ; 5-30 ;>30 \mu \mathrm{mol} / \mathrm{L})$ using the Kruskal-Wallis test , the values differed significantly

122 from each other between the three categories for both Group 1 and Group 2 (Chi-square= 41.064;

123 degrees of freedom $=2 ; \mathrm{p}<0.001$ for Group 1; Chi-square $=40.38$; degrees of freedom $=2, \mathrm{p}$

$124<0.001$ for Group 2). 


\section{Discussion}

126 The main purpose of this study was to evaluate the accuracy of the SNAP-BAT for classification

127 of bile acid concentrations as normal or increased. The accuracy of a measurement system is

128 defined as the degree of closeness to the true value. Similar to the method comparison study by

129 the manufacturer, the enzymatic chemistry analyzer used at our institution was considered the

130 gold standard, or reference method, for determination of the true value. ${ }^{7}$ To minimize variables,

131 serum samples were frozen and stored after collection so that all testing could be performed on

132 the same day, immediately after thawing. Bile acids can be stored frozen at $-20^{\circ} \mathrm{C}$ for at least 3

133 months with little variability. ${ }^{1,12}$

134 To avoid pseudoreplication, statistical analysis was compared twice, combining results from dogs

135 with a single sample and the preprandial or postprandial results of dogs with paired samples.

136 Feeding status of dogs with single samples was not critical to data analysis, since each patient

137 served as its own control. Results of statistical analysis from both groups were similar. A single

138 reference range of 0 to $20 \mu \mathrm{mols} / \mathrm{L}$ was used for both groups to account for variations in interictal

139 gallbladder activity: results of $20 \%$ of preprandial samples are reportedly greater than their paired

140 postprandial sample because of spontaneous gall bladder contraction. ${ }^{15}$

141 There are limited methods available for statistically comparing semiquantitative to fully

142 quantitative data. Because one method reports discrete data, while the other reports data on a

143 continuous scale, a medically applicable cutoff value must be used in order to determine

144 agreement between methods ( $20 \mu \mathrm{mols} / \mathrm{L}$ inthis case). The $\kappa$ index, used as the statistical method

145 of assessing agreement in the current study, varied between 0.786 and 0.819 , confirming a

146 substantial to near perfect level of agreement between the enzymatic chemistry analyzer and the

147 SNAP-BAT, based on statistical standards. ${ }^{8}$ The overall sensitivity of the SNAP-BAT is $100 \%$;

148 specificity was slightly less, ranging from $78.6 \%-83.9 \%$. Because of its high sensitivity, the 
149 SNAP-BAT will be useful for detecting liver dysfunction in affected dogs. However, it will yield

150 more false positive results than enzymatic analysis; thus, healthy dogs could be falsely diagnosed

151 with abnormal liver function. In dogs with a SNAP-BAT result $>20 \mu \mathrm{mol} / \mathrm{L}$, repeating the test

152 with an enzymatic chemistry analyzer would be therefor be necessary to verify the presence of

153 liver dysfunction.

154 Agreement between the SNAP-BAT and chemistry analyzer was further evaluated using two 155 categories $(\leq 20 \mu \mathrm{mol} / \mathrm{L}$ and $>20)$, and three categories $(<5 ; 5-30 ;>30)$. The latter categorization 156 was chosen to reflect the semi-quantitative values given by the SNAP-BAT. The results indicated 157 that values differed significantly between categories, whether there were two or three categories.

158 This further supports the strong agreement between enzymatic and SNAP-BAT results and 159 suggests that this agreement is not dependent on how the data is categorized. ${ }^{15}$

160 Based on in-house studies, the manufacturer reported an overall 95.2\% agreement between the 161 reference method and the SNAP-BAT in dogs and cats. ${ }^{7}$ Although these authors did not report 162 sensitivity, specificity, or statistical analysis of agreement, these results are similar to the results 163 reported in the present study. Furthermore, a recent study confirmed that there were no significant

164 differences in serum bile acid concentrations, as measured by a standard chemistry analyzer, 165 among laboratories. ${ }^{11}$ If methodology is consistent, it is likely that SNAP-BAT results would be 166 similarly consistent with chemistry results from multiple institutions. It was beyond the scope of 167 this study to determine accuracy of the SNAP-BAT for the diagnosis of liver disease based on 168 results of liver biopsies, since this information was not always known or recorded in each case.

169 A secondary purpose of this study was to determine precision of the SNAP-BAT. Precision, or 170 repeatability, of a measurement system is the degree to which repeated measurements under 171 unchanged conditions show the same results. The present study attempted to determine precision 172 of the SNAP-BAT by evaluation of intra-assay variability. Precision of the enzymatic method (the 
173 method used for most in-house chemistry analyzers) for determination of total serum bile acids

174 has been reported to be satisfactory. ${ }^{13}$ In the study reported here, intra-assay variability of the

175 SNAP-BAT was minimal, although the results could not be interpreted statistically because three

176 of the five samples had a uniform distribution. As noted above, the samples with a uniform

177 distribution all had values $>30 \mu \mathrm{mol} / \mathrm{L}$, whereas the two samples that exhibited intra-assay

178 variability had values between 5 and $30 \mu \mathrm{mol} / \mathrm{L}$ and therefore were fully quantified. This

179 variability is expected because of the large range of values that can be classified as $>30 \mu \mathrm{mol} / \mathrm{L}$.

180 Ideally, samples used for this portion of the study should have had more variance: for example,

181 samples with values of less than $5 \mu \mathrm{mol} / \mathrm{L}$ should have been pooled into a single sample, and

182 samples with values greater than $30 \mu \mathrm{mol} / \mathrm{L}$ should have been pooled into a single sample.

183 However, this was not possible since the SNAP-BAT and enzymatic chemistry values of the

184 sample were not known prior to thawing the samples.

185 Sample dilution is recommended for precision analysis, and use of diluent for that purpose has

186 been reported for SNAP T4 tests in dogs and cats and for SNAP IgG testing in foals. ${ }^{14,15}$ Dilution

187 is not recommended by the manufacturer for SNAP-BAT, however. In the early stages of the

188 project we attempted dilution of samples with enzymatic results $>30 \mu \mathrm{mol} / \mathrm{L}$ using a variety of

189 diluents, including saline, sterile water, plasma, and the manufacturer's diluent. Analysis of all of

190 these diluted samples resulted in an error message from the SNAP-BAT Reader or in no change

191 in the result as compared with the undiluted sample. Therefore, we do not recommend attempting

192 to further quantify results greater than $30 \mu \mathrm{mol} / \mathrm{L}$ using the SNAP-BAT by means of dilution.

193 Although the SNAP-BAT is highly accurate as a semi-quantitative test, it has several limitations.

194 The SNAP-BAT does not quantify any result less than $5 \mu \mathrm{mol} / \mathrm{L}$ or greater than $30 \mu \mathrm{mol} / \mathrm{L}$.

195 Quantification of bile acid concentrations greater than $30 \mu \mathrm{mol} / \mathrm{L}$ may be useful for

196 differentiating the etiology of the liver dysfunction. Congenital portosystemic shunts (CPSS) and 
197 PVH-MVD are related conditions in which there is a malformation of the macroscopic or

198 microscopic portal circulation, respectively. ${ }^{2,16}$ Because treatment and prognosis for the two

199 conditions vary significantly, it is critical to differentiate them. ${ }^{17,18}$ Definitive differentiation

200 between the two conditions can only be achieved by combining liver biopsy results with the

201 results of nuclear scintigraphy or other advanced imaging; however, recent data suggests that bile

202 acid results can be used to assist in distinguishing the conditions. In one study, pre- and post-

203 prandial concentrations in dogs with PVH-MVD were found to be significantly lower (41 \pm 57

$204 \mu \mathrm{mol} / \mathrm{L}$ and $92.7 \pm 37.3 \mu \mathrm{mol} / \mathrm{L}$, respectively) than in dogs with CPSS $(159 \pm 103 \mu \mathrm{mol} / \mathrm{L}$ and

$205250 \pm 148 \mu \mathrm{mol} / \mathrm{L}$, respectively). ${ }^{17}$ Of $21 \mathrm{dogs}$ diagnosed with PVH-MVD at University of

206 Tennessee (based on appropriate histologic changes and lack of shunting on nuclear

207 scintigraphy), preprandial and/or postprandial SBA were increased compared to the reference

208 range in 20/21 dogs; however, preprandial SBA were $<75 \mu \mathrm{mol} / \mathrm{L}$ in $89 \%$ of these dogs, and post-

209 prandial SBA were $<75 \mu \mathrm{mol} / \mathrm{L}$ in $81 \%$. In 100 dogs diagnosed with CPSS from 2003 to 2009 at

210 the University of Tennessee, $95 \%$ of dogs had preprandial or postprandial SBA $>50 \mu \mathrm{mol} / \mathrm{L}$;

$21193 \%$ had preprandial or postprandial SBA $>75 \mu \mathrm{mol} / \mathrm{L}$; and $76 \%$ had preprandial or postprandial

$212 \mathrm{SBA}>100 \mu \mathrm{mol} / \mathrm{L}$ (RT Hodshon, unpublished data). A fully quantitative test would be more

213 useful for differentiating between CPSS and PVH-MVD.

214 Other limitations of the SNAP-BAT include the inability to run more than 2 samples concurrently

215 and the need to purchase the SNAP Reader to interpret the results. The SNAP Reader is required

216 because, unlike the 3DX and 4DX SNAP tests, the colorimetric change for measurement of

217 SNAP-BAT cannot be visually interpreted. Benefits of the SNAP-BAT include ease of use and

218 fast, immediate, precise and highly sensitive results.

219 The study itself had several limitations. The population that provided samples was skewed

220 toward dogs with increased bile acid concentrations, since samples were obtained from dogs 
221 undergoing bile acid testing because of suspected liver disease. This resulted in a lack of normal

222 distribution to our population. The effects of serum hemolysis and lipemia, which have been

223 previously shown to falsely decrease and increase bile acid concentrations, respectively, as

224 measured by enzymatic assay ${ }^{1,19}$, were also not directly assessed in this study.

\section{Conclusion}

226 The SNAP-BAT has a substantial to near perfect level of agreement to the enzymatic chemistry

227 analyzer, is easy to use, yields immediate results, and has little intra-assay variability, particularly

228 for results greater than $30 \mu \mathrm{mol} / \mathrm{L}$. While highly sensitive, it may yield false positive results

229 compared to the enzymatic chemistry analyzer. Additionally, it is not able to quantify values

230 greater than $30 \mu \mathrm{mol} / \mathrm{L}$, limiting its utility in determining severity of liver disease.

\section{Footnotes}

a IDEXX Laboratories, Inc., Sacramento, CA 95605

233

b Cobas 501c from Roche Diagnostics, Indianapolis, IN 46225

234

c Clay Adams Brand Compact II Centrifuge, Becton Dickinson Primary Care Diagnostics,

$235 \quad$ Sparks, MD 21152

236 d Diazyme Laboratories, Poway, CA 92064

\section{References}

238 1. Jensen AL. 1991. Evaluation of fasting and postprandial total serum bile acid concentration in 239 dogs with hepatobiliary disorders. Zentralbl Veterinarmed A 38:247-54. 
240 2. Winkler JT,Bohling MW, Tillson DM, Wright JC, Ballagas AJ 2003. Portosystemic shunts:

241 diagnosis, prognosis, and treatment of 64 cases (1993-2001). Journal of American Animal

242 Hospital Association 39:169-85.

243 3. Center SA, Baldwin BH, de Lahunta A, Dietze AE, Tennant BC 1985. Evaluation of serum bile 244 acid concentrations for the diagnosis of portosystemic venous anomalies in the dog and cat.

245 Journal of American Veterinary Medical Association 186:1090-4.

246 4. Meyer DJ. 1986. Liver function tests in dogs with portosystemic shunts: measurement of 247 serum bile acid concentration. Journal of American Veterinary Medical Association 188: 168-9.

248 5. Gerritzen-Bruning MJ, van den Ingh TS, Rothuizen J. 2006. Diagnostic value of fasting plasma 249 ammonia and bile acid concentrations in the identification of portosystemic shunting in dogs.

250 Journal of Veterinary Internal Medicine 20:13-9.

251 6. Center SA, Baldwin BH, Erb HN, Tennant BC 1985. Bile acid concentrations in the diagnosis 252 of hepatobiliary disease in the dog. Journal of American Veterinary Medical Association 187(9): 253 935-40.

254 7. Holbrook L, Roth-Johnson L, Sheldon K. 2005. IDEXX SNAP Bile Acids: Method 255 comparison study. IDEXX Laboratories Inc.

256 8. Landis JK, Koch GG. 1977. The measurement of observer agreement for categorical data. 257 Biometrics 33(1): 159- 74.

258 9. Cohen JA. 1960. Coefficient of agreement for nominal scales. Educational and Psychological 259 Measurement 20: 37-46.

260 10. Center SA. 1993. Serum bile acids in companion animal medicine. Veterinary Clinics of 261 North America: Small Animal Practice 23:625.

262 11. Nanfelt M, Macphail C, Kennedy KC, Eickhoff J 2012. Interlaboratory agreement and 263 handling variability for consistency of results in paired serum bile acid assays. Journal of 264 American Animal Hospital Association 48(1): 25-30.

265 12. Olsson T. 1988. Serum bile acids in cattle: diurnal variations and variations due to stage of 266 lactation. Journal of Veterinary Internal Medicine 35(6): 467-72.

267 13. Tobiasson P, Kallberg M. 1980. Evaluation of a commercial enzymatic method for the 268 determination of total serum bile acids. Annuals of Clinical Biochemistry 17(6): 301-6.

269 14. Kemppainen RJ, Birchfield JR. 2006. Measurements of total thyroxine concentration in 270 serum from dogs and cats by use of various methods. American Journal of Veterinary Research $27167: 259-65$. 
272 15. Pusterla N, Pusteria JB, Spier SJ, Puget B, Watson JL2002. Evaluation of the SNAP Foal IgG 273 test for the semiquantitative measurement of immunoglobulin $\mathrm{G}$ in foals. Veterinary Record

274 151:258-60.

275 16. Berent AC. Tobias KM. 2009. Portosystemic vascular anomalies. Veterinary Clinics of North 276 America 39(3): 513-41.

277 17. Allen L, Stobie D, Mauldin GN, Baer KE Clinicopathologic features of dogs with hepatic 278 microvascular dysplasia with or without portosystemic shunts: 42 cases (1991-1996). 1999.

279 Journal of American Veterinary Medical Association 214:218-20.

280 18. Christiansen JS, Hottinger HA, Allen L, Phillips L, Aronson LR2000. Hepatic Microvascular 281 Dysplasia in Dogs: A retrospective study of 24 cases (1987- 1995). Journal of American Animal 282 Hospital Association 36: 385-9.

283

284 19. Solter PF, Hoffmann WE, Hoffman JW. 1992. Evaluation of an automated serum bile acids 285 assay and the effect of bilirubin, hemoglobin, and lipid on the apparent bile acids yield. 286 Veterinary Clinical Pathology 21(4):114-8. 


\section{Table 1 (on next page)}

Intra-assay variability for SNAP-BAT in umoles/L 
1 Table 1: Intra-assay variability for SNAP-BAT in $\mu$ moles/L

\begin{tabular}{|l|r|r|r|r|r|} 
& & \multicolumn{3}{|c|}{ Pooled Samples } & \\
\hline & 1 & 2 & 3 & 4 & 5 \\
\hline & & & & & \\
$\begin{array}{l}\text { Chemistr } \\
\text { y analyzer } \\
\text { value }\end{array}$ & 87 & 97 & 8 & 10 & 67 \\
\hline SNAP 1 & $>30$ & $>30$ & 8 & 11 & $>30$ \\
\hline SNAP 2 & $>30$ & $>30$ & 10 & 17 & $>30$ \\
\hline SNAP 3 & $>30$ & $>30$ & 7 & 13 & $>30$ \\
\hline SNAP 4 & $>30$ & $>30$ & 10 & 8 & $>30$ \\
\hline SNAP 5 & $>30$ & $>30$ & 12 & $<5$ & $>30$ \\
\hline SNAP 6 & $>30$ & $>30$ & $<5$ & 12 & $>30$ \\
\hline SNAP 7 & $>30$ & $>30$ & $<5$ & 10 & $>30$ \\
\hline SNAP 8 & $>30$ & $>30$ & $<5$ & 6 & $>30$ \\
\hline SNAP 9 & $>30$ & $>30$ & 9 & 10 & $>30$ \\
\hline SNAP 10 & $>30$ & $>30$ & 7 & 11 & $>30$ \\
\hline
\end{tabular}

2

3

4

5

6

7

8

9

10

11

12

13

14

15

16

17

18

19

20

21

22

23

24 


\section{Table 2 (on next page)}

Agreement of Group 1 enzymatic chemistry analysis and SNAP-BAT for results categorized as $>20 \mu \mathrm{moles} / \mathrm{L}$ or $\leq 20 \mu \mathrm{mol} / \mathrm{L}$ 
1

2 Table 2: Agreement of Group 1 enzymatic chemistry analysis and SNAP-BAT for results

3 categorized as $>20 \mu$ moles/L or $\leq 20 \mu \mathrm{mol} / \mathrm{L}$

4

\begin{tabular}{|c|c|c|c|c|c|}
\hline & & & SNAP v & ues* & \\
\hline & & & $>20$ & $\leq 20$ & Total \\
\hline $\begin{array}{l}\text { Chemistry } \\
\text { Values* }\end{array}$ & & $\begin{array}{l}\text { Count } \\
\% \text { within category }\end{array}$ & $\begin{array}{l}28 \\
100.0 \%\end{array}$ & $0 \%$ & $\begin{array}{l}28 \\
100.0 \%\end{array}$ \\
\hline & $\leq 20$ & $\begin{array}{l}\text { Count } \\
\% \text { within category }\end{array}$ & $21.4 \%$ & $\begin{array}{l}22 \\
78.6 \%\end{array}$ & $\begin{array}{l}28 \\
100.0 \%\end{array}$ \\
\hline Total & & $\begin{array}{l}\text { Count } \\
\% \text { within category }\end{array}$ & $60.7 \%$ & 32 & $\begin{array}{l}56 \\
100.0 \%\end{array}$ \\
\hline
\end{tabular}

5

$6 *$ Results are in $\mu$ moles $/ \mathrm{L}$. 


\section{Table 3 (on next page)}

Agreement of Group 2 enzymatic chemistry analysis and SNAP-BAT for results categorized as $>20 \mu \mathrm{moles} / \mathrm{L}$ or $\leq 20 \mu \mathrm{mol} / \mathrm{L}$ 
1 Table 3: Agreement of Group 2 enzymatic chemistry analysis and SNAP-BAT for results

2 categorized as $>20 \mu \mathrm{moles} / \mathrm{L}$ or $\leq 20 \mu \mathrm{mol} / \mathrm{L}$

3

4

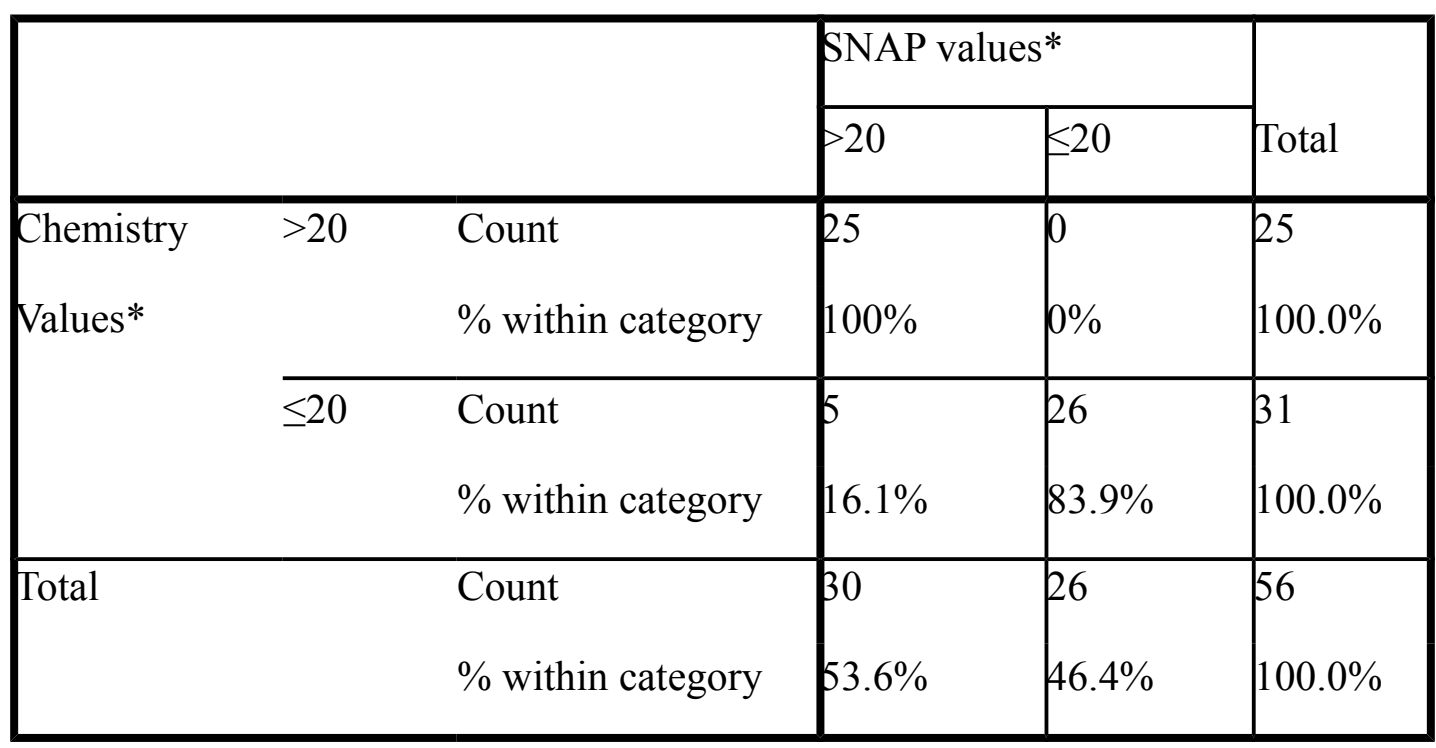

5

$6 *$ Results are in $\mu$ moles/L.

7

8

9

10

11

12

13

14

15

16

17

18

19

20

21

22

23

24

25

26

27

28 


\section{Table 4(on next page)}

Table 4: Comparisons of level of agreement and standard error between the enzymatic chemistry analyzer and SNAP-BAT for Groups 1 and 2 
1 Table 4: Comparisons of level of agreement and standard error between the enzymatic chemistry

2 analyzer and SNAP-BAT for Groups 1 and 2

3

4

\begin{tabular}{|l|l|l|}
\hline & $\begin{array}{l}* \mathrm{k} \\
\text { index }\end{array}$ & SE \\
\hline Group 1 & $\begin{array}{l}0.78 \\
6\end{array}$ & $\begin{array}{l}0.08 \\
1\end{array}$ \\
\hline Group 2 & $\begin{array}{l}0.81 \\
9\end{array}$ & $\begin{array}{l}0.07 \\
6\end{array}$ \\
\hline
\end{tabular}

5

6

7

8

9 * Interpretation of $\mathrm{k}$ index: 0 : no agreement; 0-0.2: slight agreement; $0.21-0.40$ :

10 fair agreement; 0.41-0.60: moderate agreement; 0.61-0.80: substantial agreement;

11 .0.8: almost perfect agreement ${ }^{8} ; \mathrm{SE}=$ Standard error

12

13

14

15

16

17

18 